\title{
A MULTI-AGENT SYSTEM BASED ON NATURAL LANGUAGE PROCESSING USING COLLECTIVE USER KNOWLEDGE-BASE AND GPS DATABASES
}

\author{
M. Muntean And A. Donea
}

Abstract. Nowadays, Human-Machines interfaces are improved by Natural Language Processing (NLP) techniques in order to reduce the users tasks. The proposed paper describes the implementation of an intelligent Multi-Agent System that receives requests from NLP module and consults its Collective User KnowledgeBase and GPS Database in order to provide accurate information to the user.

2010 Mathematics Subject Classification: 68T50, 68T42, 68T35.

Keywords: Natural Language Processing, Intelligent Agents, Knowledge-Base.

\section{INTRODUCTION}

Natural Language Processing (NLP) is used to improve Human Machine Interface features by understanding humans questions and giving adequate responses to them. When NLP is integrated in Intelligent Agents, it establishes the communication between man and agent in order to help the agent to better understand the human speech and to better recognize the human writing.

In paper [1], the authors propose an intelligent chatbot system in order to improve the communication between humans and machines in the travel domain. The proposed agent takes into account the Restricted Boltzmann Machine (RBM) with Collaborative Filtering proposals in order to better design the user preferences and to model collective user knowledge base for future conversations.

Other approaches [2] propose a Personal Assistant that analyses the words of the question and consults its database in order to give the proper responses. The proposed system can be used in any smart home in order to assist the living environment.

Dinus Intelligent Assistance Chatbot was proposed in [3] to interact with students and give information about University admission services. The application uses knowledge gained from machine learning approaches. 
M. Muntean and A. Donea - A Multi-Agent System ...

In mobile learning, hybrid cooperative agents were used for multilanguage textto-speech processing [4]. The authors of [4] propose to integrate a Multi-Language ontology for better results.

Our proposal is to use Natural Language Processing in order to take the user request in his natural language and to process and send it to our system agents: GPS Agent, Expert System Agent, Database Agent and Notification Agent. The Natural Language Agent permanently interacts with Collective User Knowledge-Base for giving accurate answers to the users.

\section{Natural Language Processing and Intelligent Agents. Design SYSTEM FEATURES}

The user communicates with Natural Language Agent through device sensors, keyboard and screen. AIML (Artificial Intelligence Mark-up Language) [5], [6] is defined as a processor that queries the knowledge-base and invokes heuristic search methods implemented in autonomous functions. The user can discuss with other users or execute commands for Android or for GPS sensor using his natural language (Figure $1)$.
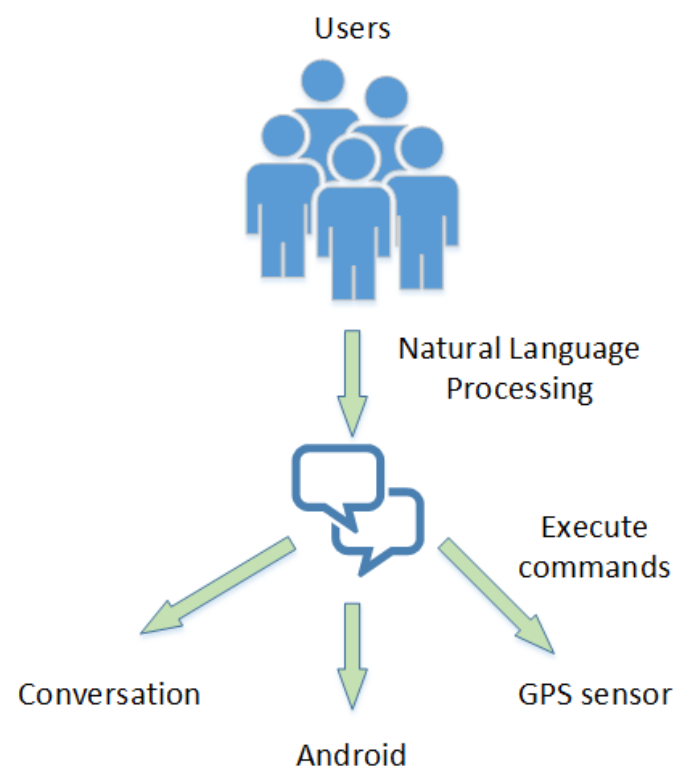

Figure 1: Natural Language Processing component design.

The developed platform can support a large number of users who communicate 
with intelligent agents to execute actions. The agents were implemented using Jade Framework [7].

In this paper we propose a Multi-Agent System that performs multiple tasks (Figure 2): receive the user requests in his natural language and compute them (Natural Language Agent), receive the user coordinates from GPS sensor (GPS Agent) in order to monitor the user route and to assist him in finding certain locations, fire rules from the Collective Knowledge-Base system (Expert System Agent) in order to answer to user questions, insert the user events into a database and use this information to assist other users (Database Agent), monitor users and the platform (Notification Agent) and facilitate the exchange messages between users (User Agent) [8].

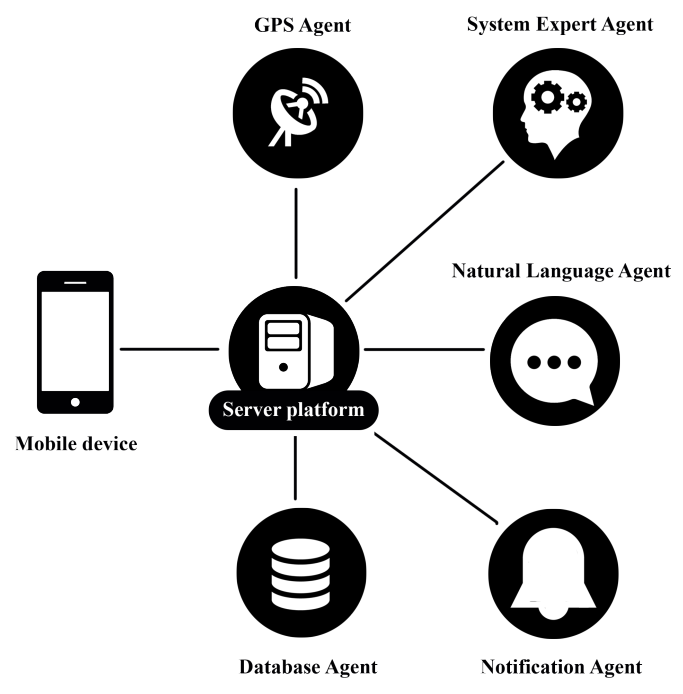

Figure 2: Design of Multi-Agent System.

The intelligent agents are hosted on a server platform that contains also the database, the knowledge-base, and the mobile device application that allows the connection of other agents.

Another advantage of the proposed system is that users can communicate with each other via the platform and send messages or get information about agents activities or about specific locations. The platform is also a social network that stores a database with information about registered users. 
M. Muntean and A. Donea - A Multi-Agent System ...

\section{Evaluating the proposed architecture}

AIML files contain data necessary to build the knowledge-base with facts and actions designed to create the conversation with users. Each user can create his own intelligent agent and can teach him words or improve his knowledge-base vocabulary. Figure 3 describes how knowledge is stored in AIML files. It is noted that each stored predicate refers to a list of sentences that are sent to the user via the agent. The search in AIML files is based on regular expressions and formal languages.

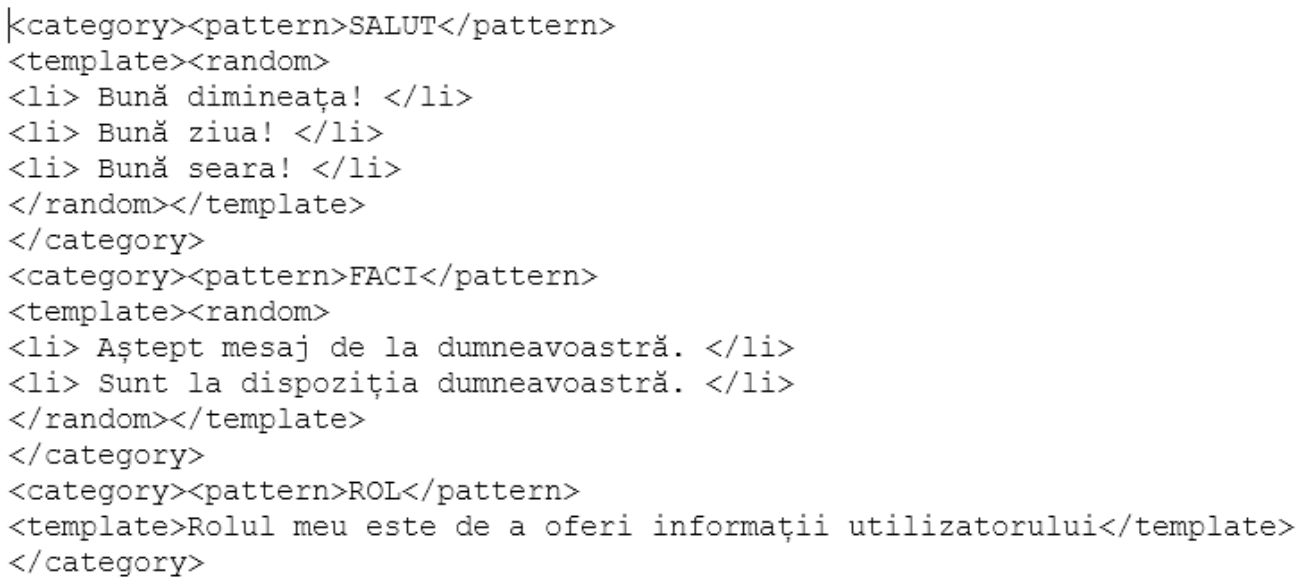

Figure 3: Sample of AIML code.

Each user has his own agent that communicates with intelligent agents through ACLMessage. The main Human Machine interface contains all the elements and options necessary to manage the database, users and agents (Figure 4). The platform also contains an interface for User - Agent conversation. The user can search information about users, history of platform activities, and intelligent agents actions.

In order to perform travel assistance, Natural Language Agent based on AIML and Expert System Agent will search for user geographic position. The user uses natural language in order to call GPS sensor which takes his coordinates (latitude and longitude) and computes the distance to an interest point. The obtained data is sent to the expert system, that analyses the information and converts the results back into natural language. The User Agent has access to the phone's calendar, helping the assistant to perform different tasks based only on phones sensors (Figure 5 and Figure 6).

Because the GPS sensor did not accurately read the actual data and the application did not perform distance computing with a high success rate, we have implemented in AIML a function that computes the exact location with one circle 
M. Muntean and A. Donea - A Multi-Agent System ...

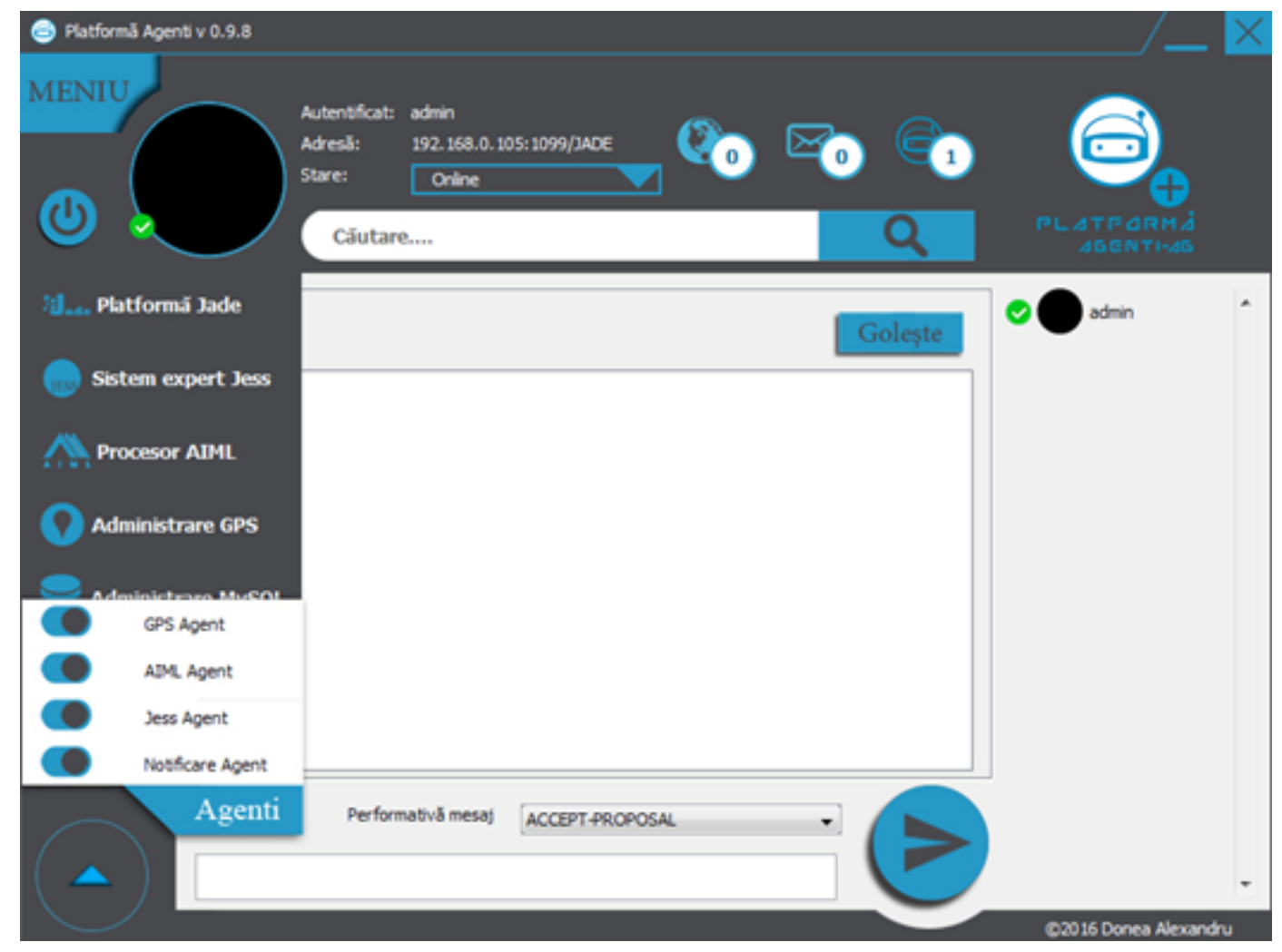

Figure 4: The main Human Machine interface.

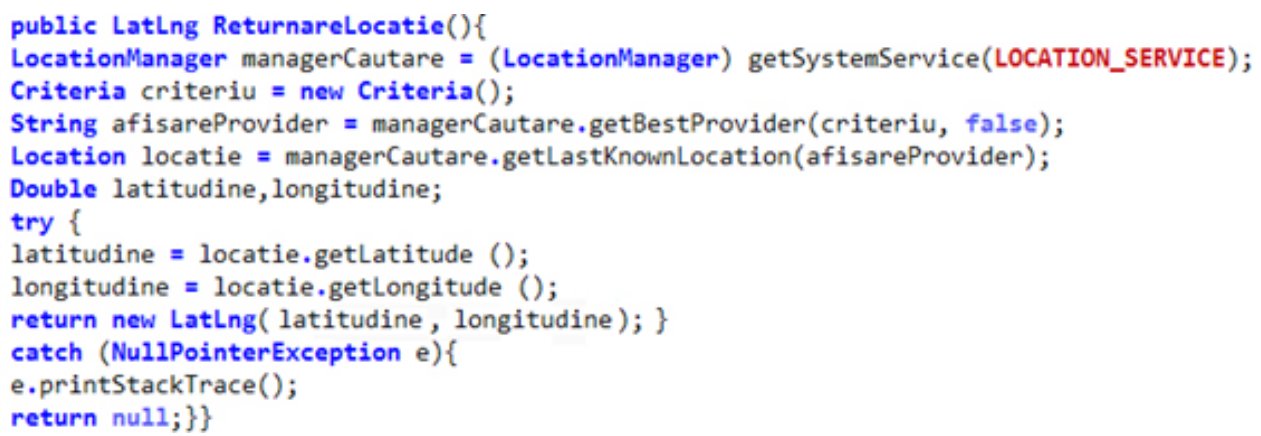

Figure 5: Computation of user geographic position.

with a radius of 1.5 kilometers around the desired location. In this way, we can avoid transmitting wrong data to the user considering the nearest interest points. 
M. Muntean and A. Donea - A Multi-Agent System ...
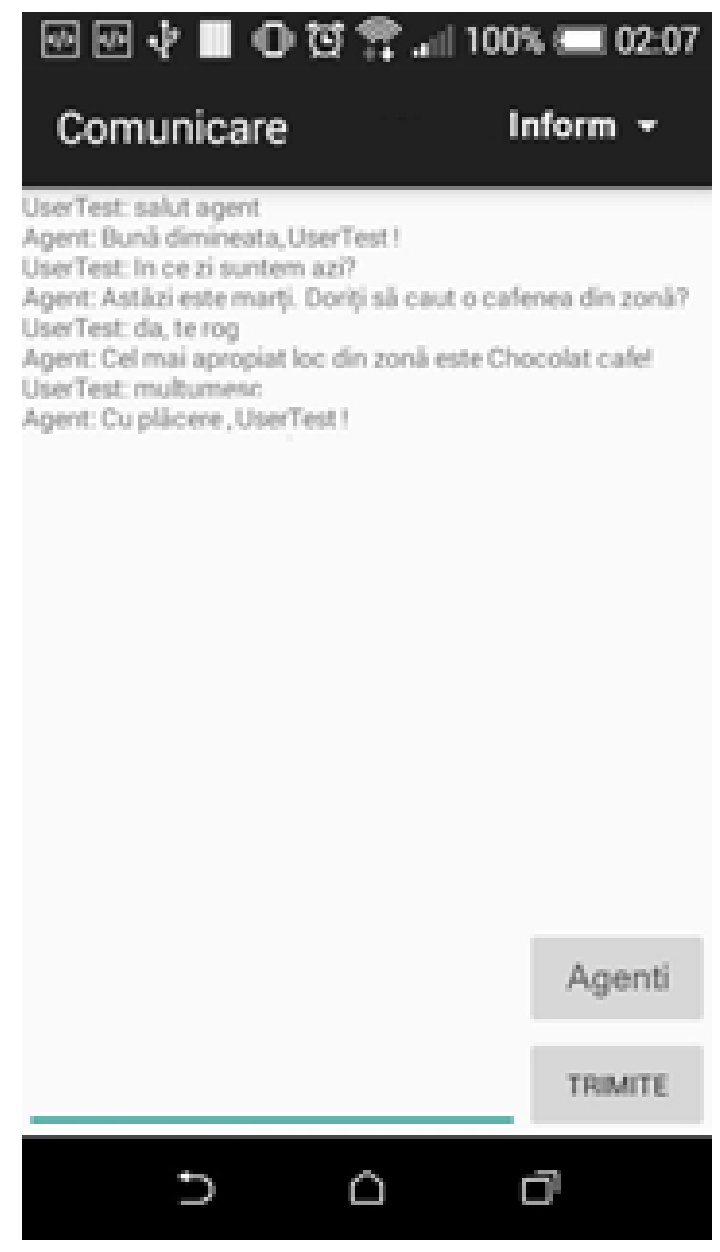

Figure 6: Experimental results using Android.

\section{Conclusions}

In this paper we proposed a Multi-Agent System that performs multiple tasks in order to assist users in travel domain. The proposed system contains an AIML module that has the ability to learn and store in memory a wide range of data sorted by categories. The designed agents assist the learning process and store the learned data using the XML language.

Due to the AIML processor, the intelligent agent is able to learn and interact with other users on the same knowledge-base, in order to improve user interactions and to permanently update the Collective User Knowledge-Base. 
M. Muntean and A. Donea - A Multi-Agent System ...

\section{REFERENCES}

[1] A. Argal, S. Gupta, A. Modi, P. Pandey, S. Shim and C. Choo, Intelligent travel chatbot for predictive recommendation in echo platform, 2018 IEEE 8th Annual Computing and Communication Workshop and Conference (CCWC), Las Vegas, NV, 2018, pp. 176-183.

[2] A. Spournias et al., Experimental Evaluation of a Novel Personal Assistant in Greek Language for Ambient Assisted Living Environments employing home robots, 2018 South-Eastern European Design Automation, Computer Engineering, Computer Networks and Society Media Conference, Kastoria, Greece, 2018, pp. 1-9.

[3] H. Agus Santoso et al., Dinus Intelligent Assistance (DINA) Chatbot for University Admission Services, 2018 International Seminar on Application for Technology of Information and Communication, Semarang, Indonesia, 2018, pp. 417-423.

[4] M. Abdelkefi and I. Kallel, Conversational agent for mobile-learning: A review and a proposal of a multilanguage text-to-speech agent, MobiSpeech, 2016 IEEE Tenth International Conference on Research Challenges in Information Science (RCIS), Grenoble, 2016, pp. 1-6.

[5] R. S. Wallace, The Elements of AIML Style, ALICE A. I. Foundation, Inc. March 28, 2003.

[6] AIML 2.0 Reference, http://callmom.pandorabots.com/static/reference/

[7] JAVA Agent DEvelopment Framework Reference, http://jade.tilab.com/

[8] M. V. Muntean and A. Donea, A hybrid intelligent agent based expert system for GPS databases, 2016 8th International Conference on Electronics, Computers and Artificial Intelligence (ECAI), Ploiesti, 2016, pp. 1-4.

Maria Muntean

Sciences and Engineering Department, 1 Decembrie 1918 University of Alba Iulia Alba Iulia, Romania email:mmuntean@uab.ro

Alexandru Donea

Sciences and Engineering Department, 1 Decembrie 1918 University of Alba Iulia Alba Iulia, Romania 